\title{
Las tecnologías de la información y la comunicación (TIC) en la Universidad Francisco de Paula Santander
}

Jhon Franklin Espinosa Castro ${ }^{1}$.

\section{Resumen}

Las tecnologías de información y comunicación (TIC), hoy en día están estableciendo un cambio en el ser humano, especialmente en el sistema educativo universitario en el proceso de enseñanza y aprendizaje, donde se debe utilizar y aplicar con eficacia. Para ayudar a los estudiantes adquirir las habilidades necesaria en agrupan los elementos y las técnicas utilizadas en el tratamiento y la transmisión de las informaciones, principalmente de informática, internet y telecomunicaciones.

Teniendo en cuenta lo anterior, en la Universidad Francisco de Paula Santander no se encontró ningún registro acerca de las tecnologías de la información y la comunicación (TIC). Debió, a que no se establecen investigaciones, e igualmente los estudiantes no tienen conocimiento en la utilización y aplicación, dentro de las diferentes carreras que establecieron información. Debió, que el 73\% de los estudiantes encuestados, utiliza el internet de la UFPS para realizar diferentes actividades.

Además, acceden al servicio utilizando un portátil en las zonas verdes, cubículos, salones o en los corredores de los edificios, en especial las zonas verdes, porque es el lugar que presenta una mejor cobertura. Asimismo, 436 estudiantes de 1000 establecen una calificación de 3 en una escala de 1 a 5. Donde la comunidad universitaria utiliza el servicio de internet en chat, redes sociales, música y juegos.

Al mismo tiempo, se espera que de esta investigación, propongan alternativas para mejorar la calidad de la enseñanza y aprendizaje, ya que los estudiantes necesitan conocer las ventajas que aportan las TIC para trabajar con éxito los avances tecnológicos.

Palabras claves. Tecnología, informática, internet y telecomunicaciones.

\section{Abstract}

The information and communication technologies (ICT), today are establishing a change in humans, especially in the university education system in the process of teaching and learning where to use and apply effectively. To help students acquire the skills necessary to gather the elements and techniques used in the processing and transmission of information, especially information technology, internet and telecommunications.

Given the above, the University Francisco de Paula Santander was not found any records about the technologies of information and communication technologies (ICT). Due, not to establishing research, and also students are not knowledgeable in the use and implementation within the different careers that established information. He must, that 73\% of students surveyed used the Internet for UFPS for different activities.

Furthermore, accessing the service using a laptop in the green spaces, cubicles, rooms or in corridors of buildings, especially the green areas, because it is the place that has better coverage. Also, 436 students of 1000 established a score of 3 on a scale of 1 to 5 . Where 
the university community uses the Internet chat service, social networking, music and games.

At the same time, it is expected that this research, propose alternatives to improve the quality of teaching and learning, as students need to know the advantages provided by ICT to successfully work with technological advances.

Keywords. Technology, computers, internet and telecommunications.

\section{Introducción}

$\mathrm{L}$ as tecnologías de la información y la comunicación son las principales herramientas, utilizadas en diversas áreas, lugares y estancias, con fin de aplicar, utilizar y aprovechar adecuadamente el avance en la tecnología que tenemos a nuestro alcance con los diversos e innovadores equipos que hacen que se facilite y agilice la información y las comunicaciones, aumentando la conectividad social a nivel regional, nacional y mundial, disminuyendo factores como el tiempo, distancias y costos.

Además, ofrecen un enorme potencial para transformar la educación. Es decir, modernizar y renovar sus sistemas educativos para establecer mejor calidad. Por esa razón, Los docentes necesitan estar preparados para empoderar a los estudiantes con las ventajas que les aportan las TIC.

En la educación presencial a distancia o virtual, igualmente, los docentes deben posean las competencias y los recursos necesarios en materia de TIC y que puedan enseñar de manera eficaz las asignaturas exigidas, integrando al mismo tiempo en su enseñanza conceptos y habilidades de estas. Las simulaciones interactivas, los recursos educativos digitales y abiertos, los instrumentos sofisticados de recolección y análisis de datos son algunos de los muchos recursos que permiten a los docentes ofrecer a sus estudiantes posibilidades, antes inimaginables, para asimilar conceptos.

En este sentido, esta investigación se lleva a cabo con la intención de conocer la aceptación y el conocimiento de la comunidad universitaria de UFPS, en informática, internet y telecomunicaciones. Para tener una mejor visión del vínculo que hay entre las TIC y los estudiantes, para establecer cómo los estudiantes manejan las TIC en su aprendizaje, en la búsqueda de información e investigación.

\section{Materiales y métodos}

La herramienta que se utilizó y permitió la obtención de la información es un cuestionario, que está orientado a conocer la aceptación y el conocimiento de la comunidad universitaria en las TIC. De ahí que la investigación es descriptiva, donde los resultados se presentarán en tablas de distribución de frecuencias y representaciones gráficas, para así exponer los ítems que se analizaron. Además, recolectada la información, el proceso de registro, de codificación de los datos obtenidos y análisis, se utilizó el programa Microsoft Office Excel.

\section{Resultados y discusión}

Figura 1. Género.

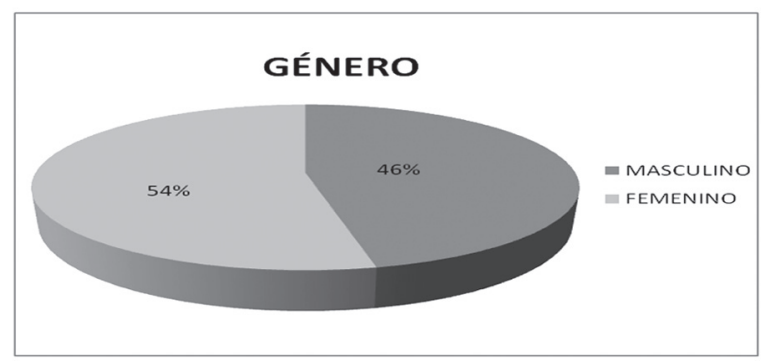

Como se observa en la figura 1 . De un porcentaje del $100 \%$ de estudiantes, el $54 \%$ son del género femenino y el $46 \%$ masculino. 
Figura 2. Edad.

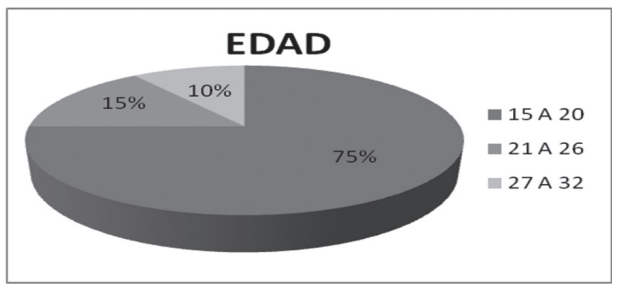

Como se observa en la figura 2. Las edades de los educandos oscilan entre 15 a 32 años, además el $75 \%$ de los estudiantes tienen entre 15 a 20 años. Es decir, alumnos que están cursando la mitad sus estudios de pregrado.

Figura 3. Semestre académico que cursa

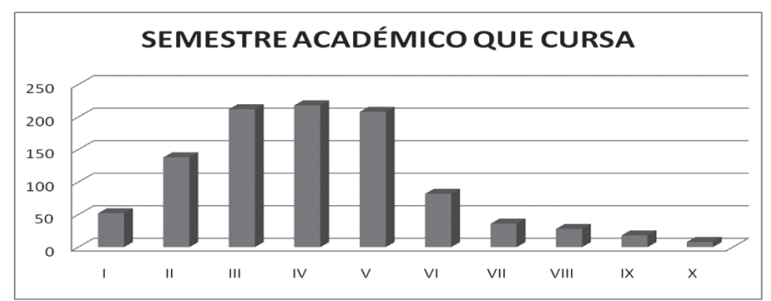

Como se observa en la figura 3. Los educandos que aportaron información para esta investigación están entre II y VI semestre académico. Además, representa el 90\% de los estudiantes de diferentes carreras.

Figura 4. Conocimientos en las tecnologías de la información y comunicación.

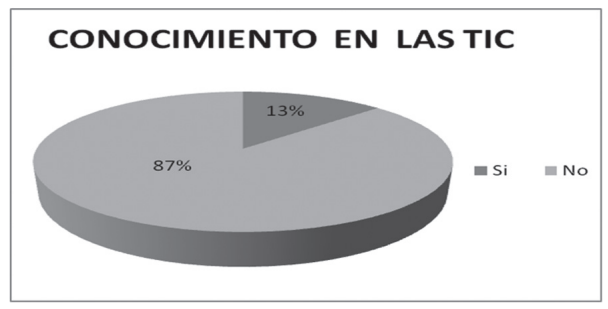

Como se observa en la figura 4. El 87\% los estudiantes no tiene conocimiento en las TIC, en el proceso de enseñanza - aprendizaje.

Figura 5. Tipo de tecnología móvil que utiliza.

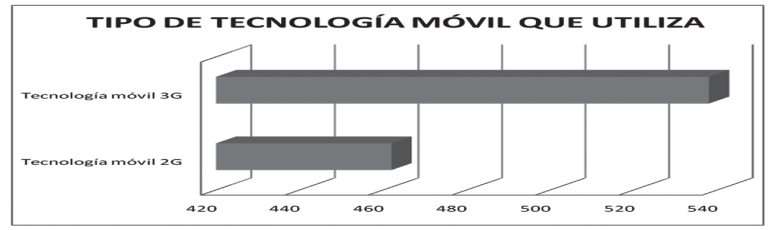

Como se observa en la figura 5. Se establece que de 1000 estudiantes, hay 538 que utilizan la tecnología 3G, que proporcionan la posibilidad de transferir tanto voz y datos (una llamada telefónica o video llamada) y datos no-voz (como la descarga de programas, intercambio de email, y mensajería instantánea).

Figura 6. Conocimiento en el manejo de un portátil.

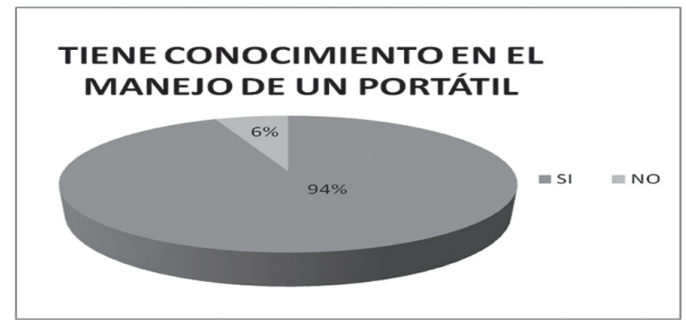

Como se observa en la figura 6. El 94\% de los estudiantes tienen un conocimiento en el manejo de un portátil, estableciendo una ventaja para el uso y aplicaciones de NTIC.

Figura 7. Utiliza el servicio de internet de la UFPS.

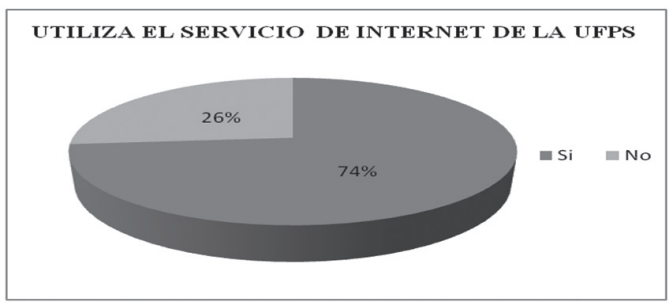

Como se observa en la figura 7. El 74\% utilizan el servicio de internet de la universidad, a través de un portátil, la sala de informática de la biblioteca o equipos celulares con tecnología 3G.

Figura 8. Utiliza un portátil para acceder a la conexión de internet en la UFPS.

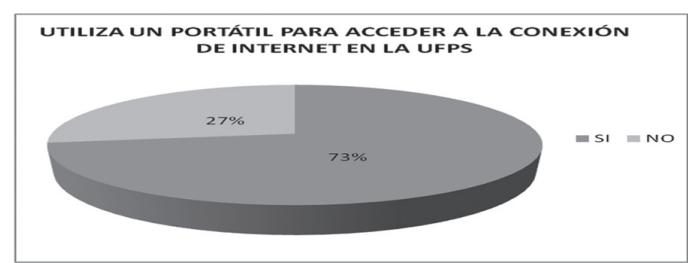

Como se observa en la figura 8. Un total de 726 estudiantes utilizan un computador portátil de propiedad de un familiar, amigo o propio, en diferentes espacios de la universidad para realizar diferentes actividades. Además, los 274 alumnos restantes utilizan el internet de la sala 
de informática de la biblioteca o equipos celulares con tecnología 3G.

Tabla 1. Donde accede a internet en la UFPS utilizando un portátil.

\begin{tabular}{|l|r|}
\hline \multicolumn{2}{|c|}{ DONDE ACCEDE A INTERNET EN LA UFPS } \\
\hline En los corredores de los edificios & 76 \\
\hline En las aulas de clase & 187 \\
\hline Biblioteca & 132 \\
\hline En alguna oficina, dependencia o facultad & 12 \\
\hline Sala de proyección & 32 \\
\hline Zonas verdes o cubiculos & 782 \\
\hline Parqueadero de carros & 46 \\
\hline Canchas deportivas & 28 \\
\hline
\end{tabular}

Como se observa en la tabla 1. La mayoría de los estudiantes acceden al servicio de internet de la universidad, utilizando un portátil en las en zonas verdes o cubículos, para realizar diferentes actividades entre las que se encuentran consultas, servicios de chat, redes sociales, mensajería instantánea y música. Además, en aula de clase, para exponer, realizar una consulta individual o grupal, verificar o profundizar un contenido. Ver tabla 2.

A continuación, se analizará los servicios de internet que utilizan los estudiantes, a través de un portátil en las en zonas verdes o cubículos, en las aulas de clase y biblioteca, teniendo en cuenta que se podía seleccionar más de una opción.

Tabla 2. Servicios que utiliza de internet

\begin{tabular}{|l|r|}
\hline \multicolumn{2}{|c|}{ SERVICIOS QUE UTILIZA DE INTERNET } \\
\hline Buscadores & 95 \\
\hline Navegadores & 42 \\
\hline Páginas web especificas & 547 \\
\hline Portales especificos & 362 \\
\hline Juegos & 125 \\
\hline Música & 672 \\
\hline Servicios de chat & 796 \\
\hline Correo electrónico instantáneo & 875 \\
\hline Páginas para hacer curso virtuales & 35 \\
\hline Redes Sociales & 932 \\
\hline Páginas para descargar programas & 56 \\
\hline
\end{tabular}

Como se observa en la tabla 2. La utilización de redes sociales, correo electrónico instantáneo, servicios de chat y música, son los servicio más utilizados por los estudiantes a través de internet en las instalaciones de la universidad. De acuerdo a lo anterior, el servicio de internet se utiliza para el entretenimiento, comunicación y páginas web específicas para consulta.

Figura 9. Calificación del servicio de internet en la UFPS

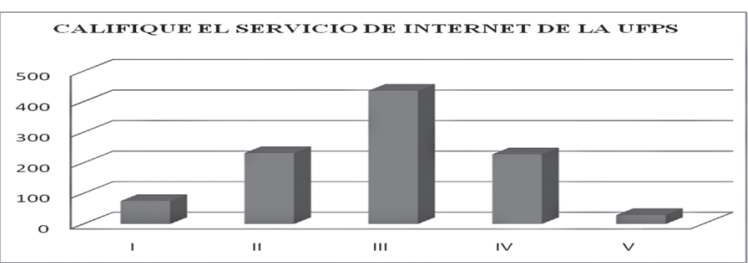

Como se observa en la figura 9. Un total de 436 estudiantes de 1000 , especifican que el servicio de internet no es el más adecuado por su velocidad de navegación y descarga, dándole una calificación de 3 en una escala de 1 a 5.

Tabla 3. Nivel de conocimiento en informática.

\begin{tabular}{|l|r|r|r|r|}
\hline \multirow{2}{*}{$\begin{array}{c}\text { NIVEL DE CONOCIMIENTO } \\
\text { EN INFORMÁTICA }\end{array}$} & \multicolumn{4}{c|}{ Nivel } \\
\cline { 2 - 5 } & Nulo & Básico & Medio & Alto \\
\hline Sistemas operativos (Windows o Linux) & 126 & 723 & 129 & 22 \\
\hline Procesador de textos (Word o otros) & 84 & 176 & 568 & 172 \\
\hline Bases de datos (Access o otros) & 973 & 16 & 9 & 2 \\
\hline Hojas de cálculo(Excel o otros) & 756 & 139 & 76 & 29 \\
\hline Presentaciones (PowerPoint o otros) & 15 & 136 & 302 & 547 \\
\hline $\begin{array}{l}\text { Internet (Navegadores, e-mail, chat, música, } \\
\text { juegos o otros) }\end{array}$ & 12 & 49 & 116 & 823 \\
\hline $\begin{array}{l}\text { Software educativo (Cursos de inglés, } \\
\text { informáticos o otros) }\end{array}$ & 978 & 17 & 2 & 3 \\
\hline Programas de animación(Flash o otros) & 983 & 12 & 4 & 1 \\
\hline $\begin{array}{l}\text { Programas de tratamiento de } \\
\text { imágenes(Corel draw o otros) }\end{array}$ & 959 & 25 & 12 & 4 \\
\hline Programación(Visual Basic o otros) & 886 & 67 & 39 & 8 \\
\hline Programas en 3D(Autocad o otros) & 989 & 7 & 3 & 1 \\
\hline
\end{tabular}

Como se observa en la tabla 3. Los estudiantes tienen un conocimiento alto en el manejo de internet, presentaciones y procesadores de texto, por lo tanto, carecen de un conocimiento en el manejo de paquetes informáticos.

Tabla 4. La dificultad en utilizar las tecnologías de la información y comunicación.

\begin{tabular}{|l|r|}
\hline \multicolumn{2}{|c|}{ LA DIFICULTADES EN UTILIZAR LAS NTIC } \\
\hline Disponibilidad de tiempo en capacitación & 136 \\
\hline No utiliza lo último en tecnologia y aplicaciones & 72 \\
\hline $\begin{array}{l}\text { Poca aceptación de las NTIC en el proceso de } \\
\text { enseñanza-aprendizaje }\end{array}$ & 82 \\
\hline $\begin{array}{l}\text { No existen canales de información que establezcan } \\
\text { su utilización y aplicación }\end{array}$ & 710 \\
\hline
\end{tabular}


Como se observa en la tabla 4. Se puede establecer que la principal dificultad, es que no existen canales de información que establezcan su utilización y aplicación, en el proceso de enseñanzaaprendizaje, en el conocimiento de las TIC.

\section{Conclusiones}

Teniendo en cuenta la investigación realizada, se puede establecer que un porcentaje aceptable de los estudiantes que suministraron información, han utilizado alguna vez, las tecnologías de la información y la comunicación. Debió, que estas tecnologías están presentes en la vida cotidiana de los estudiantes, haciéndole más fácil su aprendizaje en las distintas áreas del conocimiento.

Además, se puede determinar que el $73 \%$ de los estudiantes encuestados, utiliza el internet de la UFPS para realizar diferentes actividades. Además, acceden al servicio utilizando un portátil en las zonas verdes, cubículos, salones o en los corredores de los edificios, en especial las zonas verdes, por que es el lugar que presenta una mejor cobertura. Asimismo, 436 estudiantes de 1000 establecen una calificación de 3 en una escala de 1 a 5. Donde la comunidad universitaria utiliza el servicio de internet en chat, redes sociales, música y juegos. Sin tener en cuenta, que el internet es una red de conocimientos.

Se recomienda, capacitar a los profesores en el manejo y aplicaciones de las TIC, para el desarrollo de material didáctico educativo, para el proceso de enseñanza -aprendizaje en cada una de las aéreas del conocimiento.

También, proporcionar canales de información a través de los administrativos, estudiantes y profesores, que se encargue de divulgar y asesorar de manera constante la información acerca de los adelantos tecnológicos, para así lograr un acercamiento al alumnado y se interesen.

Por lo tanto, las tecnologías de información y comunicación, en el sistema educativo universitario, enriquecen y complementan la enseñanza, el aprendizaje, la investigación y las labores administrativas de las universidades.
Igualmente, una retroalimentación de los estudiantes por medio de sus experiencias, simplificar la comunicación e innovar.

Por esas razones, las TIC requiere de una infraestructura, accesos seguros, desempeño adecuado a nuestras necesidades y un apoyo técnico siempre disponible. Para lograr cumplir esta promesa de las TIC necesitamos disponer de un PETI (Plan Estratégico de Tecnología de Información) que permita que los recursos TIC sean confiables, innovadores y continuamente actualizados.

\section{Agradecimiento}

El autor expresa sus más sinceros agradecimientos a los estudiantes de la Universidad Francisco de Paula Santander por su colaboración en recolección de la información.

\section{Bibliografía}

Álvaro Uribe Vélez. Ley de TIC 1341 del 30 de julio de 2009. República de Colombia - Gobierno Nacional.

Pérez Marqués Graells (2008). Las TIC y sus aportaciones a la sociedad. Disponible: http:// peremarques.pangea.org/tic.htm. (Consulta: 2010, julio 1).

Wikipedia. Tecnologías de la información y la comunicación. Disponible: http: // es.wikipedia.org/wiki/Tecnolog\%C3\%ADas_ de_la_informaci \% C $3 \%$ B $3 n_{-} y_{-} l_{\text {a }}$ comunicaci\%C3\%B3n. (Consulta: 2010, julio 1).

Colombia digital. TIC en el mundo. Panorama general de las TIC y su entorno en el mundo. Disponible: http://www.colombiadigital.net/ tic-en-el-mundo/tic-el-mundo.html. (Consulta: 2010, julio 1).

Colombia aprende la red del conocimiento. Las TIC: más cerca de los docentes. Disponible: http:// www.colombiaaprende.edu.co/html/home/1592/ article-81640.html. (Consulta: 2010, julio 1). 